\title{
Oscillation and Cross-section Results from T2K
}

\author{
Stephane Zsoldos*t \\ Queen Mary University of London \\ E-mail: s.zsoldos d qmul . ac.uk
}

T2K is an experiment designed to observe neutrino oscillations with a baseline of $295 \mathrm{~km}$ across Japan from the J-PARC accelerator complex in Tokai to the Super-Kamiokande detector in Kamioka. Its main goal is to measure oscillation parameters though $v_{\mu}$ disappearance and $v_{e}$ appearance. T2K was the first experiment which published an indication of non-zero value for the mixing angle $\theta_{13}$, and recently published its first result in the search for $\mathrm{CP}$ violation in neutrino oscillations combining appearance and disappearance channels for $v / \bar{v}$ beam.

Entering the age of precision measurement of the neutrino parameters, T2K published neutrinonucleus cross-section measurements of various interaction channels at T2K off-axis (ND280) and on-axis (INGRID) near detectors.

In this talk, the latest oscillation and cross-section results are reported.

XIV International Conference on Heavy Quarks and Leptons (HQL2018)

May 27- June 1, 2018

Yamagata Terrsa, Yamagata, Japan

\footnotetext{
* Speaker.

${ }^{\dagger}$ On behalf on the T2K Collaboration
} 


\section{Introduction}

In 2015, the Nobel Prize of Physics was awarded to T. Kajita and A. McDonald, of the SuperKamiokande and SNO experiments, for "The discovery of neutrino oscillation, which shows that neutrinos have mass". This mechanism is the first direct probe of physics Beyond the Standard Model (BSM) available for the particle physics community, which is entering a new era of precision measurements of the neutrino oscillation parameters.

Neutrino oscillation depends on non-zero neutrino mass by using a mechanism highly analogous to the one in place in the quark sector, meaning that the weakly-interacting neutrino states are not eigenvectors of the Schrödinger Hamiltonian. Therefore, one can introduce a mixing matrix to change basis between these two Fock spaces:

$$
\underset{\alpha \equiv e, \mu, \tau}{\left|v_{\alpha}\right\rangle}=\sum_{i} U_{\alpha i}\left|v_{i}\right\rangle \Longleftrightarrow\left(\begin{array}{c}
v_{e} \\
v_{\mu} \\
v_{\tau}
\end{array}\right)=\left(\begin{array}{ccc}
u_{e 1} & u_{e 2} & u_{e 3} \\
u_{\mu 1} & u_{\mu 2} & u_{\mu 3} \\
u_{\tau 1} & u_{\tau 2} & u_{\tau 3}
\end{array}\right)\left(\begin{array}{c}
v_{1} \\
v_{2} \\
v_{3}
\end{array}\right)
$$

Integrating the Schrödinger equation and squaring the neutrino wavefunction lead to the oscillation probability formula. The amplitude of the oscillation becomes a function of the mixing matrix coefficient elements, and the squared-mass difference of the mass neutrino states gives the frequency of the oscillatory behaviour :

$$
\begin{aligned}
P(\alpha \rightarrow \beta)(L, E)=\delta_{\alpha \beta} & -4 \sum_{i} \sum_{j<i} \mathfrak{R}\left(U_{\alpha i} U_{\beta i}^{*} U_{\alpha j}^{*} U_{\beta j}\right) \sin ^{2}\left(1.27 \Delta m_{i j}^{2} \frac{L[m]}{E[\mathrm{MeV}]}\right) \\
& -2 \sum_{i} \sum_{j<i} \mathfrak{I}\left(U_{\alpha i} U_{\beta i}^{*} U_{\alpha j}^{*} U_{\beta j}\right) \sin \left(2.54 \Delta m_{i j}^{2} \frac{L[m]}{E[\mathrm{MeV}]}\right)
\end{aligned}
$$

The unitarity of the mixing matrix, with some clever parametrization, allows one to write the matrix element using only three parameters and one phase (with an additional two phases if the neutrino appears to be described by a Majorana spinor, but it does not matter in the case of the oscillation mechanism as these phases would cancel out), which are therefore the ones probed by neutrino oscillation experiments. This representation takes its name from the famous physicists who introduced it, who are Pontecorvo, Maki, Nakagawa and Sakata:

$$
U_{\mathrm{PMNS}}=\left(\begin{array}{ccc}
1 & 0 & 0 \\
0 & c_{23} & s_{23} \\
0 & -s_{23} & c_{23}
\end{array}\right) \times\left(\begin{array}{ccc}
c_{13} & 0 & s_{13} e^{-i \delta_{\mathrm{CP}}} \\
0 & 1 & 0 \\
-s_{13} e^{i \delta_{\mathrm{CP}}} & 0 & c_{13}
\end{array}\right) \times\left(\begin{array}{ccc}
c_{12} & s_{12} & 0 \\
-s_{12} & c_{12} & 0 \\
0 & 0 & 1
\end{array}\right)
$$

With $c_{i j} \equiv \cos \theta_{i j}, s_{i j} \equiv \sin \theta_{i j}$ and $\Delta m_{i j}^{2}=m_{i}^{2}-m_{j}^{2}$. $\delta_{C P}$ is the phase characterizing the difference in behaviour between the neutrino and its antiparticle.

\section{The T2K experiment}

The Tokai-to-Kamiokande (T2K) experiment is devoted to measuring the mixing angles and squared mass difference of the neutrino states. Initially, the experiment was designed to report 
evidence of oscillation between $v_{\mu} \rightarrow v_{e}$ flavor states (lead by the mixing angle $\theta_{13}$ ) and precise measurements of the $\left(\theta_{23},\left|\Delta m_{32}^{2}\right|\right)$ parameters. Since the publication of the non-zero value of $\theta_{13}$ [1] and the latest updates on the atmospheric sector parameters [2], T2K is leading the measurement of the $\delta_{C P}$ value. This is achievable by the ability to run with neutrino or anti-neutrino data. Fig. 1 broadly shows the key features of the T2K beam and detectors setup.

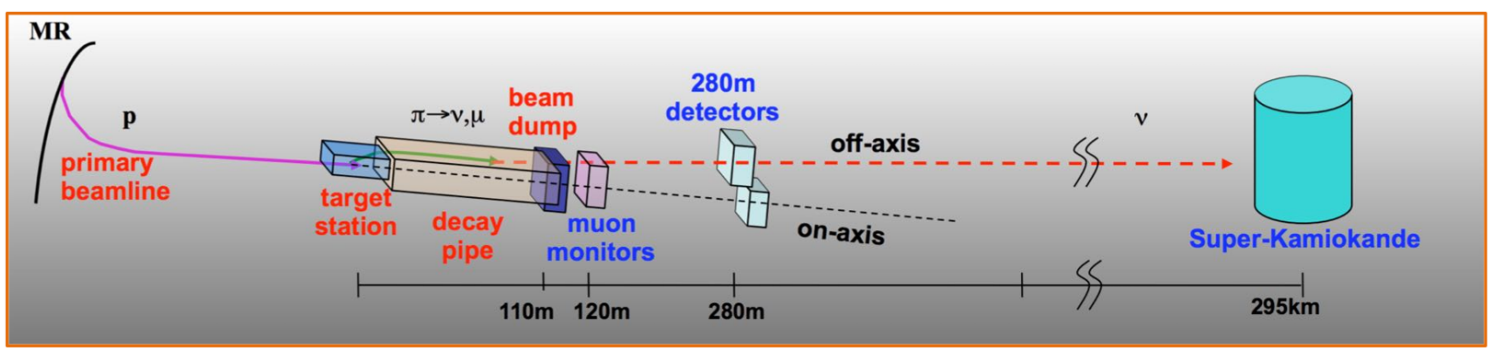

Figure 1: Schematic view of the T2K experiment: $30-\mathrm{GeV}$ protons hit a graphite target $p+C \rightarrow \pi^{ \pm}+X$, then $\pi^{ \pm}$decay and produce muon (anti)neutrinos. The near detectors (off-axis ND280 and on-axis INGRID) are situated at $280 \mathrm{~m}$, and the far detector Super-Kamiokande is $295 \mathrm{~km}$ away from the target

T2K consists of a set of detectors located in Japan and aligned in the direction of a neutrino beam produced at the J-PARC facility in Tokai, Ibaraki. This beam is generated from $30 \mathrm{GeV}$ protons hitting a graphite target producing pions and kaons focused thanks to magnetic horns. It is this magnetic field who allows the charge selection of the proton collision products, which therefore decays to a nearly pure $v_{\mu}$ or $\bar{v}_{\mu}$ beam peaked around $0.6 \mathrm{GeV}$.

$280 \mathrm{~m}$ from the beam source, two detectors are installed to monitor the beam stability day-byday looking at neutrino interaction (INGRID cf. Fig. 2) and measuring the unoscillated neutrino spectrum (ND280 cf. Fig. 3) respectively.

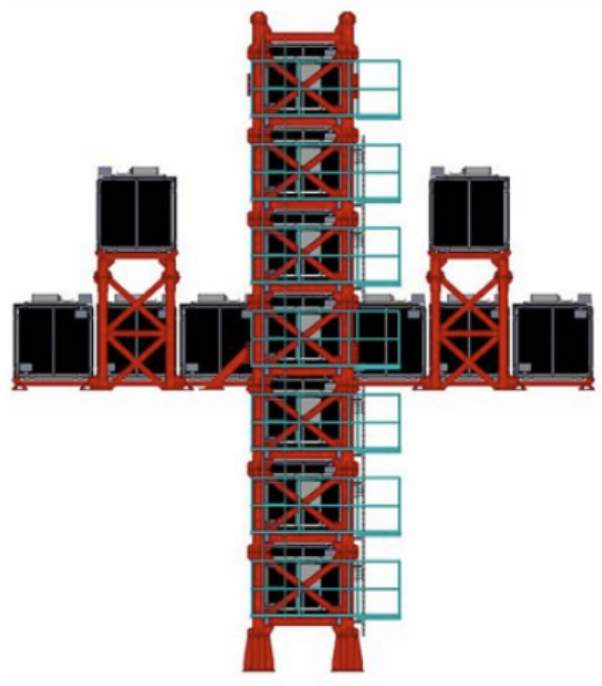

Figure 2: On-axis detector located at 280m from beam source INGRID

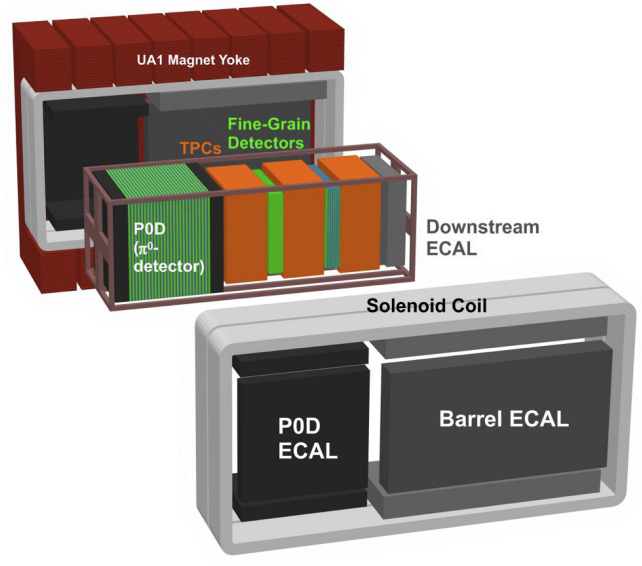

Figure 3: Off-axis detector located at $280 \mathrm{~m}$ from beam source ND280

One of the main features of the $\mathrm{T} 2 \mathrm{~K}$ experimental setup is an implementation of the so-called off-axis beam concept : due to kinematics of the $\pi^{ \pm}$decays at some small angles (with respect to 
the initial proton beam) the produced neutrinos have an energy spectrum with a rather narrow peak which can be tuned to the oscillation maximum/minimum. In T2K the value of 2.5 degrees off-axis angle is found to be the best (cf. Fig. 4) for the fixed L/E ratio ( $L=295 \mathrm{~km}$ and $E \approx 600 \mathrm{MeV}$ ). It also allows the suppression of the flux of neutrinos from the high-energy tail, neutral-current interactions which can mimic the signal events.

INGRID is made of 16 identical modules of iron and scintillator layers, where 14 are arranged in a cross centered on the beam axis and two additional modules are placed an off-axis position from the main cross to monitor the asymmetry of the beam. Another module, called the proton module, is made only of scintillator planes and is located on the beam axis, in front of the main cross [3].

The ND280 near detector is composed of a scintillator-based $\pi^{0}$ detector (P0D), three gas Time Projection Chambers (TPCs) and two Fine Grained Detectors (FGDs) surrounded by an electromagnetic calorimeter (ECal) and a $0.2 \mathrm{~T}$ magnetic field produced by the refurbished UA1/NOMAD magnet where the detector is inserted. The TPCs enable 3D tracking, particle identification and determination of momentum and charge. FGD1 and FGD2 are made of finely segmented scintillator bars used as a hydrocarbon target for neutrino interactions and allowing measurements of short tracks. FGD2 also contains water layers alternated with the scintillator layers [3].

The neutrino spectrum is again measured $295 \mathrm{~km}$ away inside the Super-Kamiokande detector, a 40m high cylindrical tank of 50ktons of ultra-pure water whose walls are covered with 11129 20" PMTs, collecting the Cherenkov light by the faint signals from incoming particles produced in neutrino interactions. Its excellent capability of distinguishing muons and electrons, consequently tagging $v_{\mu}$ and $v_{e}$ (or their corresponding antiparticles), allow T2K to carry out two kinds of measurement: disappearance of $v_{\mu}$ and appearance of $v_{e}$.

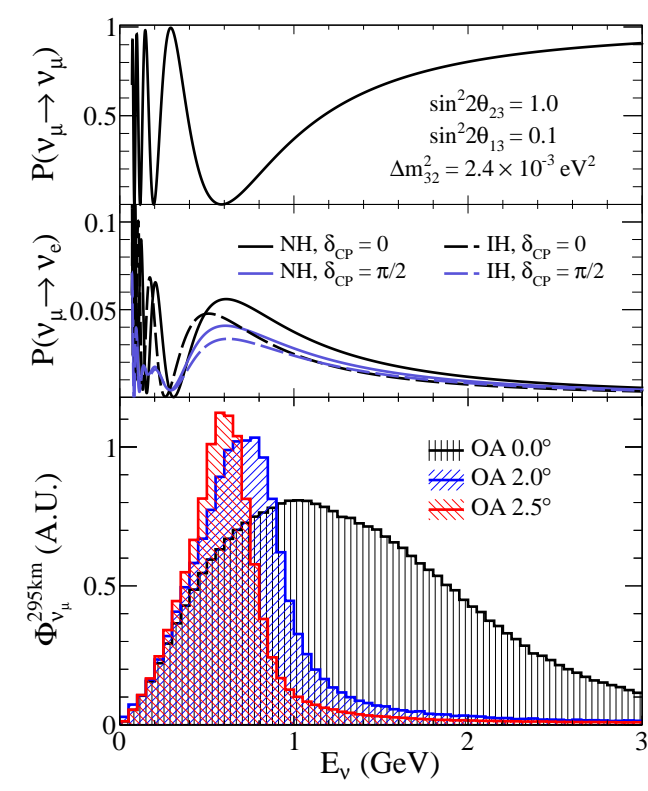

Figure 4: The oscillation probability of the $v_{\mu}$ and $v_{e}$ are overlapping with the expected neutrino spectrum measured at Super-K. It shows that the narrow flux peak is right at the dip of the $v_{\mu} \rightarrow v_{\mu}$ and the peak of $v_{\mu} \rightarrow v_{e}$. This indeed results from the optimal design in which the Super-K is at an angular offset of 2.5 degrees from the centre of the beam.

While the disappearance of $v_{\mu}$ is essential for extracting the atmospheric parameters $\left(\theta_{23},\left|\Delta m_{32}^{2}\right|\right)$, the appearance of $v_{e}$ is sensitive to $\theta_{13}$ and $\delta_{C P}$. The CP violation if it happens can manifest itself in the difference between the $v_{\mu} \rightarrow v_{e}$ and $\bar{v}_{\mu} \rightarrow \bar{v}_{e}$. In T2K, the complete three-flavor oscillation 
formulas including the matter effect with the earth density $\rho=2.6 \mathrm{~g} / \mathrm{cm}^{3}$ is used. Experimentally, with conventional $v_{\mu}$ beam, the CP violation phase can be measured by comparing $v_{\mu} \rightarrow v_{e}$ and $\bar{v}_{\mu} \rightarrow \bar{v}_{e}$ probabilities. In approximation at the maximum oscillation length of $\Delta m_{31}^{2}$, this CP asymmetry is expressed as :

$$
A_{\mathrm{CP}}=\frac{P_{v_{\mu} \rightarrow v_{e}}-P_{\bar{v}_{\mu} \rightarrow \bar{v}_{e}}}{P_{v_{e} \rightarrow v_{\mu}}-P_{\bar{v}_{e} \rightarrow \bar{v}_{\mu}}}
$$

T2K started taking data from January 2010 and up to May 2017, a total $2.23 \times 10^{21}$ Protonson-target (POT), consisting of $1.47 \times 10^{21}$ POT in neutrino-mode operation and $0.76 \times 10^{21}$ POT in antineutrino-mode operation, have been delivered to T2K detectors. This exposure is equal to $34 \%$ of fully approved T2K data set. At the time of writing these proceedings, T2K is running in anti-neutrino mode with a stable beam power of $485 \mathrm{~kW}$ and a total exposure exceeds $3 \times 10^{21}$ POT.

The T2K flux is peaked around $600 \mathrm{MeV}$ allowing to study the (anti)neutrino-nucleus scattering in an energy region where the main interaction channels are charged-current (CC) quasi elastic (CCQE), CC resonant pion production (CC-RES) and CC deep inelastic scattering (CCDIS). Since neutrinos interact with a bounded nucleon, nuclear effects alter the expected final state particle types and kinematics. Indeed, correlations between the bounded nucleons can bring to the multinucleon knock-out (also called $2 \mathrm{p} 2 \mathrm{~h}$ ). In these processes a neutrino interacts with a correlated pair of nucleons that is knocked-out from the nucleus. Another possible process is the scattering or the absorption of the final state hadrons in the nuclear environment, called final state interactions (FSI), which can also produce additional final state particles. The reconstruction of the true neutrino interaction type is limited not only by the unavoidable nuclear effects but also by detector acceptance and efficiencies. Therefore to minimize the model dependence of the measurements, is better to define experimental observables using final state inclusive topologies like $\mathrm{CC}$ interaction without pion in the final state $(\mathrm{CC} 0 \pi)$ and $\mathrm{CC}$ interaction with one pion in the final state $(\mathrm{CC} 1 \pi)$.

\section{Results}

\subsection{T2K recent cross section results}

Recently, an updated measurement of the $v_{\mu}$ CC inclusive flux integrated cross-section on ${ }^{12} \mathrm{C}$ was made using the upstream FGD as target. The previous measurement [6] had limited statistics because only forward-going muons were analysed. In the updated measurement more data $-5.7 \times 10^{21}$ POT - was added as the acceptance has been improved selecting also the high angle and backward going muons, with respect to the beam direction using the timing information between the ND280 subdetectors. Selecting particles entering the TPC, particle identification is performed (the TPC PID), while to identify particles that do not enter the TPC we are using a PID algorithm based on the ECal segments. Then, the cross section is extracted as function of the muon kinematics performing an extended binned likelihood fit. The background, which is mainly due to pions misidentified as muons, is constrained by using two control regions. The $v_{\mu}$ CC inclusive cross-section is shown in Fig. 5 in cases where the nominal Monte Carlo used as prior in the fit is NEUT (blue bands) or GENIE (black crosses). The result is then compared against NEUT, with Relativistic Fermi Gas (RFG) nuclear model and NuWro, with Local Fermi Gas (LFG). As is no- 
ticeable, both RFG and LFG overestimate the cross section in the low momentum bins, as exhibited on the bottom plots of Fig. 5 .
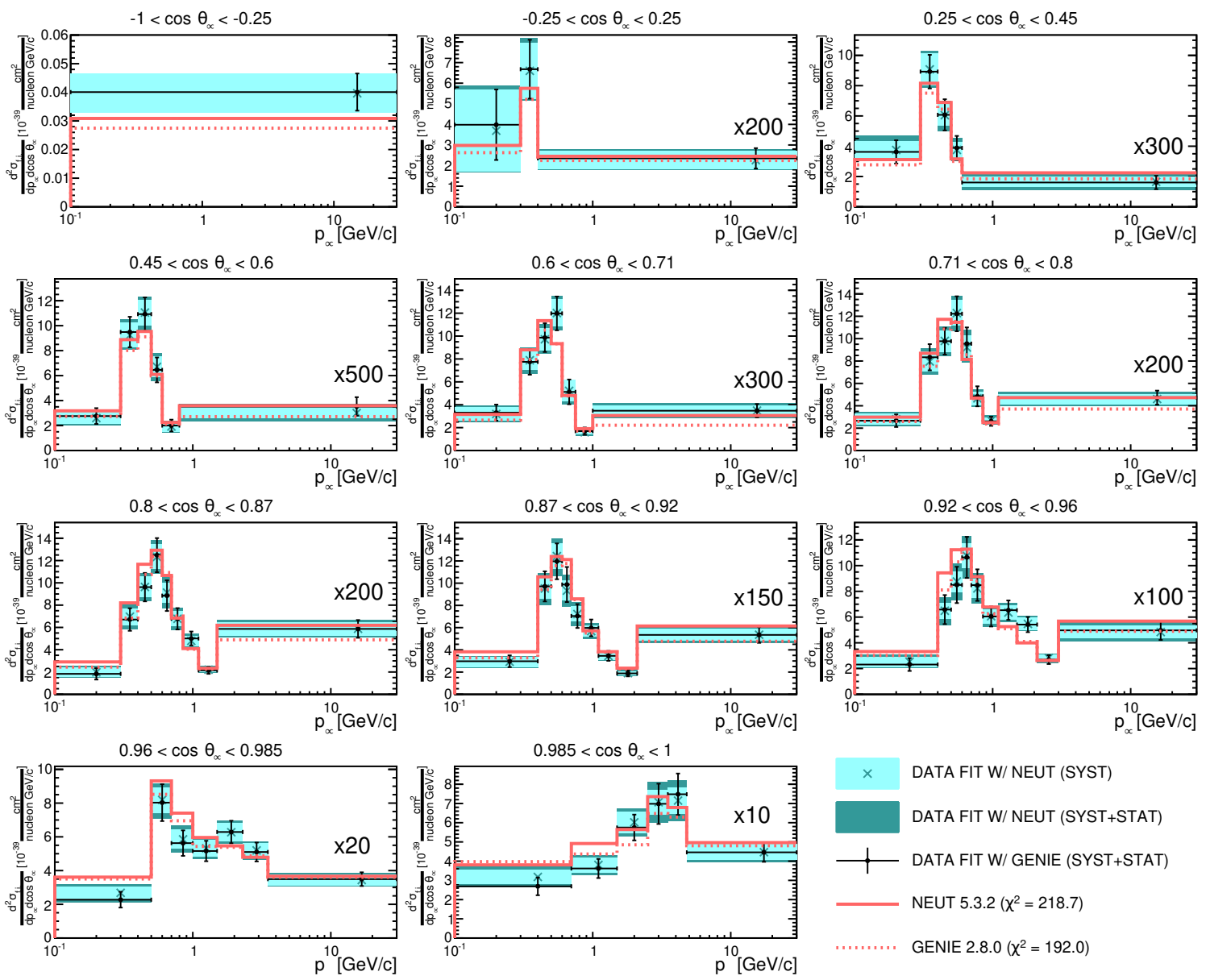

Figure 5: The flux-integrated, double-differential cross section per nucleon for NEUT (continuous red line), for GENIE (dashed red line), and the unfolded-data result using as prior either NEUT or GENIE. The bin of highest momentum is scaled by the factor shown in each plot to make it visible. $\chi^{2}$ values are computed with unfolded-data result using as prior NEUT.

\subsection{Neutrino oscillation analysis}

The neutrino oscillation parameters are estimated by comparing Monte-Carlo prediction with the measured spectrum at ND280 and Super-Kamiokande.

The neutrino fluxes are initially inferred by the combination of hadron production models, the external thin-target hadron-production data, the actual proton beam conditions, the horn currents, and the neutrino beam-axis direction measurements. At the peak energy of $600 \mathrm{MeV}$, the flux uncertainty is approximately $9 \%$, dominated by the uncertainty in hadron production data. However, its impact on estimating the neutrino oscillation parameters, given that the T2K near detector and far detector are observing data from nearly the same flux, is largely suppressed when the T2K near detector data are included. In fact, due to the convolution of both flux and neutrino interaction models on the near detector data, the data samples from ND280 are used in a manner that the 
parameters modeling the flux and interaction models are constrained simultaneously. Finally, the Super-K data along with its detector model are added into the framework of oscillation parameter extraction.

Three samples of CC interactions are selected in neutrino mode: one sample of $v_{\mu}$ and one sample of $v_{e}$ CCQE interactions, that only produce an outgoing nucleon under the Cherenkov threshold, and a charged lepton whose kinematic information is sufficient for extracting the energy of the incident neutrino. The third sample contains $v_{e} \mathrm{CC} 1 \pi^{+}$, where an electron is produced with an undetected nucleon and a charged pion, that is selected to be under the Cherenkov threshold and tagged from delayed clusters of photomultipliers hits. Assuming the reaction occurs with the production of a $\Delta^{++}$resonance decaying into a pion and nucleon. In antineutrino modes, two CCQE samples of $\bar{v}_{\mu}$ and $\bar{v}_{e}$ interactions are selected. Due to $\pi^{-}$absorption inside water, it is not possible to add the sample of $\bar{v}_{e} \mathrm{CC} 1 \pi^{-}$. These samples are shown in Fig. 6.

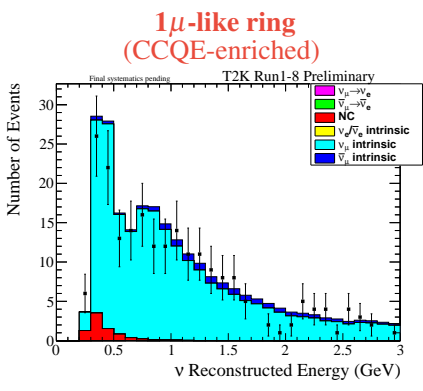

Data : $240 v_{\mu}$ (Expected: 267.8)

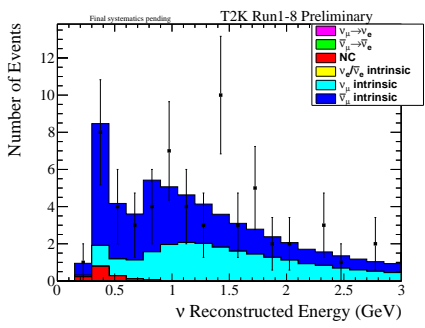

Data : $\mathbf{6 8} \bar{v}_{\mu}$ (Expected: 63.1 )

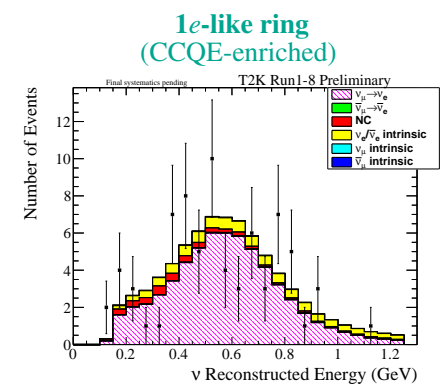

Data : $74 v_{e}$ (Expected: 73.5 )

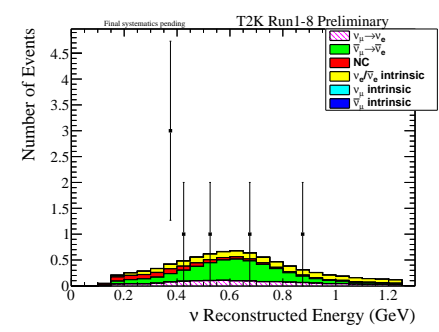

Data : $7 \bar{v}_{e}$ (Expected: 7.9$)$

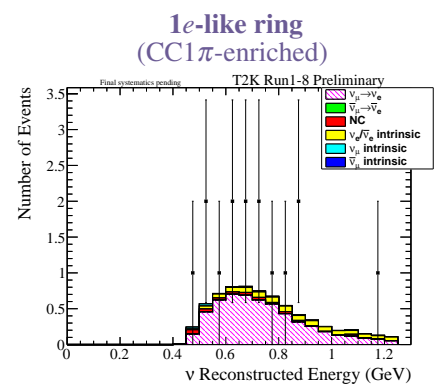

Data : $6.9 v_{e}$ (Expected : 15)

No CC $1 \pi$ in $\bar{v}$ mode due to $\pi^{-}$absorption

Figure 6: T2K far-detector data sample distributions for $v$-mode at top plots and $\bar{v}$-mode at bottom plots. Prediction of the expected events for each sample is also shown compared to the measured sample size. The topologies of the samples are indicated on the top of the figure.

Two statistical methods are used to estimate the neutrino oscillation parameters. The first is a frequentist method, that consists of first fitting the ND280 data, then propagating the obtained constraints on the flux and cross-section parameters in a covariance matrix used as prior on the fit to the Super-K data. A minimising log-likelihood method is used to estimate the value and confidence intervals of the oscillation parameters, with the Feldman-Cousins method applied on the extraction of $\delta_{C P}$ [4]. The second method relies on Bayesian statistics, performing a simultaneous analysis of the ND280 and Super-K data where the posterior probability density is sampled with a Markov Chain Monte-Carlo method in order to extract credible intervals and best-fit values of the oscillation parameters. In both cases, the likelihood is marginalised over the systematic parameters and the analysis is performed with and without the information of $\sin 2 \theta_{13}$ measured by the reactor experiments [5]. 
The best-fit values of $\sin ^{2} \theta_{23}$ are $0.526_{-0.036}^{+0.032}$ for the normal and $0.530_{-0.034}^{+0.030}$ for the inverted ordering, and that of $\left|\Delta m_{32}^{2}\right|$ are $2.463 \pm 0.065 \times 10^{-3} \mathrm{eV}^{2}$ for the normal and $2.431_{-0.064}^{+0.065} \times 10^{-3}$ $\mathrm{eV}^{2}$ for the inverted ordering (Fig. 7). The analysis including constraints from reactor experiments has also been performed and shows an excellent agreement with the T2K only result. The result is consistent with maximal $v_{\mu} \rightarrow v_{\mu}$ disappearance.

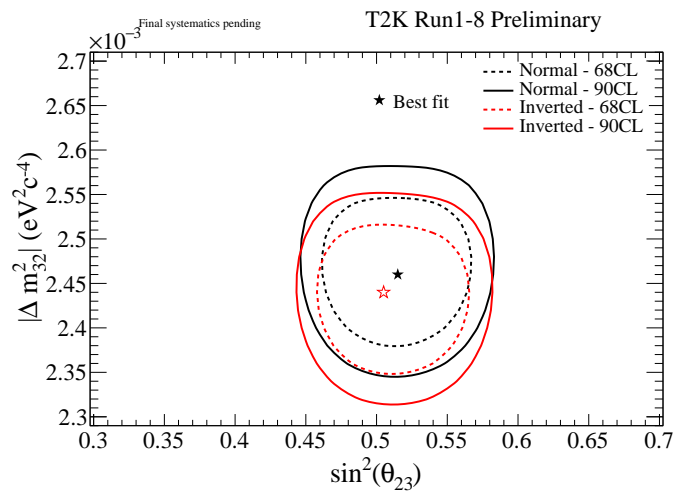

T2K Only

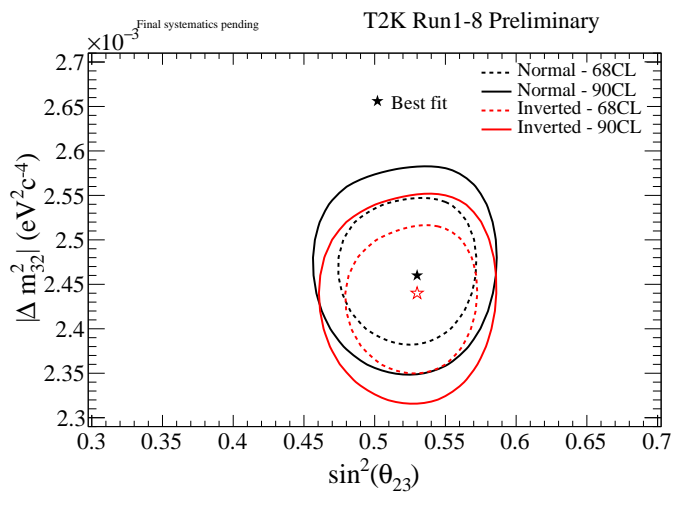

$\mathrm{T} 2 \mathrm{~K}+$ Reactor

Figure 7: $68 \%$ (dotted) and $90 \%$ (solid) confidence regions for the (( $\left.\sin ^{2} \theta_{23},\left|\Delta m_{32}^{2}\right|\right)$ parameters and best-fit values for normal (black) and inverted (red) ordering without (left) and with (right) the reactor constraints.

Fig. 8 shows the favored region on $\sin ^{2} \theta_{13}$ and $\delta_{C P}$. T2K data continue to favor the maximal disappearance. By marginalizing the likelihood over the nuisance parameters and other oscillation parameters, the resulting posterior probabilities show a moderate preference to normal mass hierarchy $(86.8 \%)$ and $\theta_{23}>\frac{\pi}{4}(78.1 \%)$. Parameter $\sin ^{2} \theta_{13}=0.0277_{-0.0047}^{+0.0054}$, measured by T2K data, is consistent with reactor measurements.

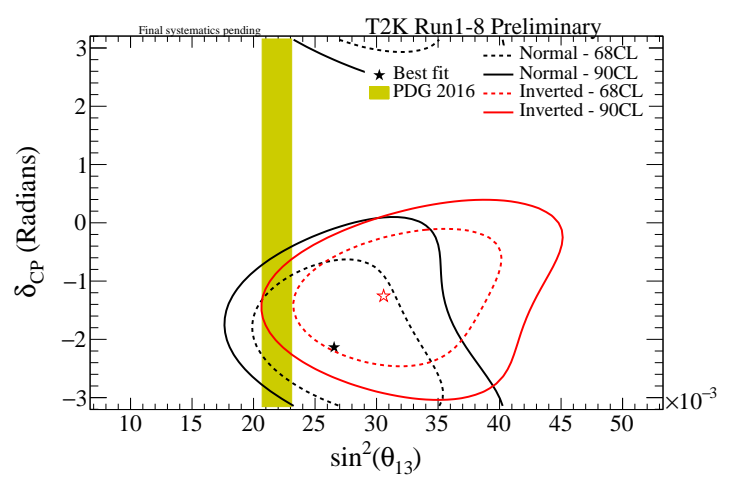

T2K Only

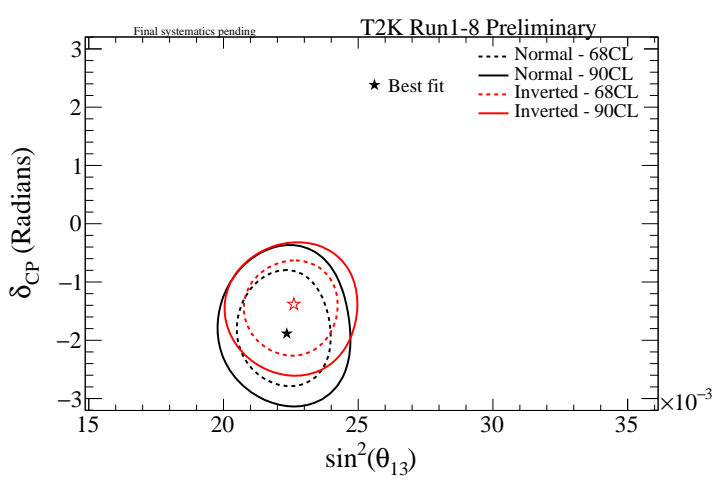

$\mathrm{T} 2 \mathrm{~K}+$ Reactor

Figure 8: $68 \%$ (dotted) and $90 \%$ (solid) confidence regions for the (( $\left.\sin ^{2} \theta_{13},|\delta C P|\right)$ parameters and best-fit values for normal (black) and inverted (red) ordering without and with the reactors constraints.

Fig. 9 shows $2 \sigma$ C.L. interval for the measured $\Delta \chi^{2}$ distribution as a function of $\delta_{C P}$ which is obtained by integrating over other parameters and taking the reactor measurement into account. The best fitted values of $\delta_{C P}$ are close to $-\pi / 2$ for both cases of mass hierarchy. The resulting intervals at $2 \sigma$ C.L. are $[-2.91,-0.60]$ for normal mass hierarchy $\left(\Delta m_{32}^{2}>0\right)$ and $[-1.54,-1.19]$ 
for inverted mass hierarchy $\left(\Delta m_{32}^{2}<0\right)$. The CP conserving values $(0$ and $\pi)$ fall outside of these intervals.
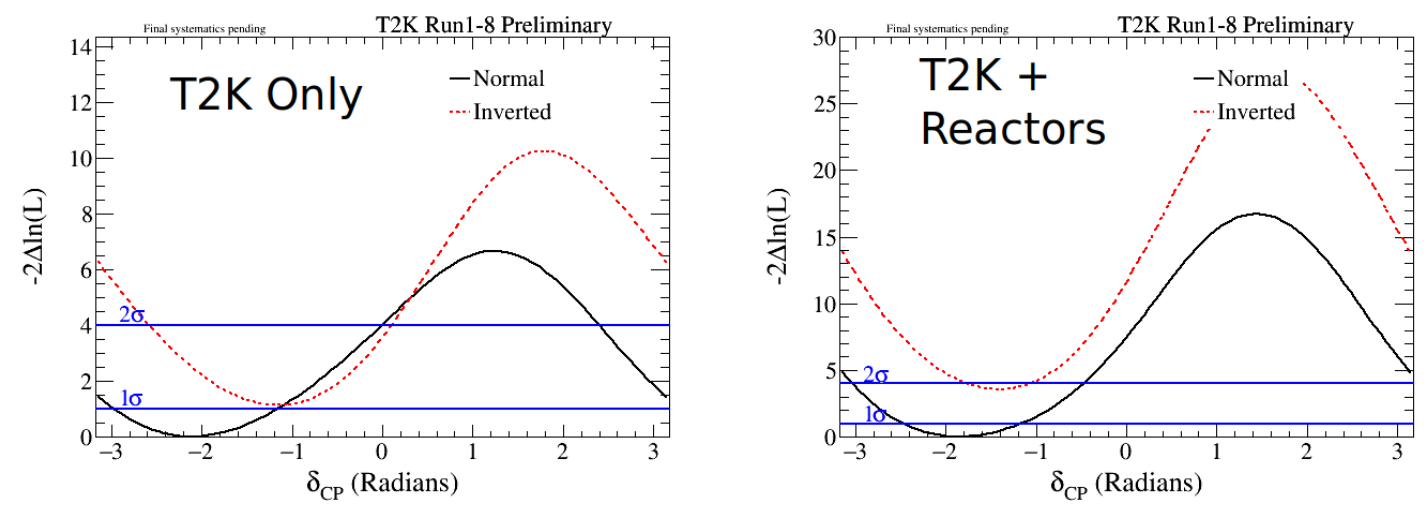

Figure 9: $2 \sigma$ confidence interval for the measured $\Delta \chi^{2}$ distribution. $\theta_{13}$ constrained by reactor measurements is used.

\subsection{T2K Prospects in the near Future}

The full approved T2K data set of, $7.8 \times 10^{21}$ POT, can be accumulated by 2021 . J-PARC aims to complete beam upgrades at $1 \mathrm{MW}$ and higher from 2021. Hyper-Kamiokande, the next generation of neutrino experiments in Japan, is expected to start operation around 2026. T2K-II, with extended operation until 2026, will collect $20 \times 10^{21}$ POT. This large amount of data along with neutrino beamline upgrade and analysis improvement make T2K(-II) physics potentials even more interesting. In particular, $3 \sigma$ or higher significance sensitivity to $\mathrm{CP}$ violation can be achieved if $\delta_{C P}$ is close to $\pi / 2$. The precision of $\Delta m_{32}^{2}$ can be $1 \%$ while that on $\theta_{23}$ is about [0.5-1.7] degrees depending on the true value. For more detail, refer to the report on the physics potential of T2KII [7]. The impact on $\delta_{C P}$ measurement from systematic error is significant and thus motivates for ND280 upgrade which is now happening actively.

\section{References}

[1] K. Abe et al. [T2K Collaboration], Phys. Rev. Lett. 112, 061802 (2014).

[2] K. Abe et al. [T2K Collaboration], Phys. Rev. D 96, 092006 (2017).

[3] K. Abe et al. [T2K Collaboration], Nucl. Instrum. Meth. A 659, 106 (2011).

[4] G. J. Feldman and R. D. Cousins, Phys. Rev., D57:3873-3889, (1998).

[5] J. Beringer et al. [Particle Data Group], Phys. Rev. D 98, 030001 (2018).

[6] K. Abe et al. [T2K Collaboration], Phys. Rev. D 87, no. 9, 092003 (2013).

[7] K. Abe et al. [T2K Collaboration], arXiv:1607.08004. 